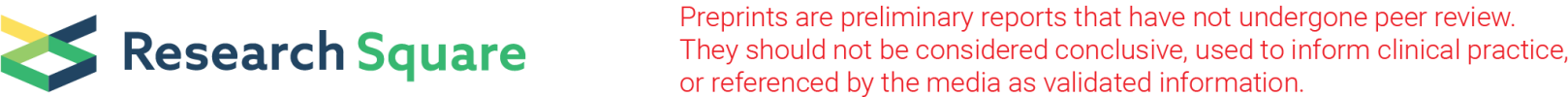

\section{"Effect of Yoga Intervention on Skeletal Muscle Linked Glucose Homeostasis in Pre-Diabetic Individuals: Study Protocol for a Randomized Controlled Trial"}

Supriya Sudhakar Bhalerao ( $\nabla$ supriya.bhalerao@gmail.com )

Bharati Vidyapeeth Deemed University https://orcid.org/0000-0003-3689-028X

Jayshree Kharche

Symbiosis International (Deemed University) (SIU)

Shubhangi Harke

Bharati Vidyapeeth Deemed University

Study protocol

Keywords: Exercise, Muscle assessment, IDRS, OGTT

Posted Date: September 13th, 2021

DOI: https://doi.org/10.21203/rs.3.rs-181905/v1

License: (c) (1) This work is licensed under a Creative Commons Attribution 4.0 International License.

Read Full License 


\section{Abstract}

Background- The pre-diabetic stage is important in terms of identification and timely management, as it is reversible. Along with other factors, insulin resistance in skeletal muscle is often considered as primary defect before development of overt hyperglycemia and diabetes. Proven to have many health benefits, Yoga has emerged as an effective measure in management of diabetes. As the Yogic postures are relatable to exercises, it was considered for improving skeletal muscle function and reverse the prediabetic stage.

Methods- The present open, randomized clinical study is planned to evaluate the effect of Yoga interventions on muscle mass, strength, endurance and flexibility which are direct or indirect indicators of fat deposition in skeletal muscles. The study will also explore association between changes in muscle quality/ functionality and glycemic control. With Ethics committee permission, 120 pre-diabetic individuals of either sex between the age group of 35-49 years with a BMI in the range of $25-35 \mathrm{~kg} / \mathrm{m}^{2}$ and family history of T2DM will be recruited in study after obtaining written informed consent. They will be randomly allocated to either Yoga or exercise group by computer generated randomized method in a 1:1 ratio. One group will receive defined Yoga interventions, while other group will receive fixed program of physical exercises. Both groups will undergo a training session for a period of 4 weeks under the supervision of a trained instructor, before 12-week study duration. The outcome measures will be parameters related to muscle quality/functionality and glucose metabolism markers.

Discussion- Our study aims to study usefulness of Yoga in prediabetic stage on skeletal muscle related glycemic control, which is novel. In addition, the study will generate awareness about pre-diabetes and provide people with active coping skills for reversing it.

Trial Registration- Clinical Trial Registry-India -CTRI/2019/05/019149, registered on: 15/05/2019

\section{Background}

Currently diabetes prevalence is rising in India. ${ }^{1}$ India ranks second in case of Pre-diabetes, which is condition occurring before diabetes and does not have any symptoms. ${ }^{2}$ Pre-diabetes stage if identified and treated properly can be reverted. ${ }^{3}$ Hence identifying and treating pre-diabetes becomes important. Physical activities can be helpful in prevention of such diseases. ${ }^{4}$

Skeletal muscle is predominant site for insulin mediated glucose uptake. Skeletal muscle linked glucose homeostasis plays a crucial role in development of Type 2 Diabetes Mellitus. Insulin resistance in skeletal muscle is often considered as primary defect before development of overt hyperglycemia and diabetes. ${ }^{5}$ The association of diabetes and muscle quality/ functionality is well established. Hence, improvement in muscle quality can be potential therapeutic target for treatment of pre-diabetes and diabetes. 
Yoga has many physical and mental health benefits. ${ }^{6}$ Yoga is said to be effective in diabetes management and is well reported in diabetic people. ${ }^{7,8}$ However, the mechanism behind this is still unknown. Physical exercise facilitates skeletal muscle glucose uptake in both insulin independent and insulin dependent ways. ${ }^{9}$ In this light, we hypothesize that Yoga intervention may also facilitate glycemic control by improving skeletal muscle function.

\section{Methods}

Study design

The present study is open, randomized clinical study.

Objectives of the study.

1. To evaluate the effect of Yoga interventions on muscle mass, strength, endurance and flexibility which are direct or indirect indicators of fat deposition in skeletal muscles.

2. To study the association between changes in muscle quality/ functionality and glycemic control.

\section{Study setting}

Recruitment of patients, biochemical investigations from serum samples, regular follow up and study documentation will be done at Interactive Research School for Health Affairs (IRSHA), Bharati Vidyapeeth Deemed University, Pune. Muscle assessment test will be carried out at Department of Physiology, Bharati Vidyapeeth Medical College, Bharati Vidyapeeth Deemed University, Pune.

\section{Sample size}

The screening process will continue until 120 pre-diabetic individuals are selected (60 for Yoga group and 60 for exercise group). This is considering an anticipated drop-out/no-show/refusal of $n=30$ to complete the study.

\section{Characteristics of study_participants}

Participants are eligible to be included in the study if the following criteria are fulfilled:

1. Individuals of either sex between the age group of 35-49 years with a BMI in the range of 25-35 $\mathrm{kg} / \mathrm{m}^{2}$

2. Individuals with family history of T2DM

3. Individuals ready to follow all procedures

Participants are excluded from the study if any of the following criteria are applicable:

1. Known cases of T2DM 
2. Individuals with known history of any other endocrinological disorders like Hypothyroidism, Aldosteronism etc.

3. Individuals with known history of medical conditions like Hypertension, joint pains, abdominal pains etc.

4. Known cases of musculoskeletal disorders like muscle dystrophy, poliomyelitis, paraplegia, hemiplegia etc.

5. Individuals with history of any surgery in past 1 year

6. Individuals deemed unfit for cardio-respiratory exercises by a physician

7. Individuals involved in any kind of vigorous or strenuous activity (such as gymnastics, aerobic exercises, dancing, swimming, jogging etc.) or any Yogic activities (Asanas, breathing exercises, meditation etc.) in last 6 months

8. Pregnant or lactating females

The Indian Diabetes Risk Score (IDRS), popularly used tool for screening of diabetes in Indian population, has been considered while developing eligibility criteria.

Interventions and Procedures-

After obtaining Institutional Ethics Committee permission, the project will be carried out in 4 steps.

Step 1: Identification of pre-diabetics through community screening

A community-based screening will be carried out for the above-mentioned eligibility criteria after providing detail information about the study procedures to the potential candidates and documenting their informed consent.

Those individuals fulfilling the eligibility criteria will be called to the study site in fasting state for estimation of Fasting plasma glucose (FBG) and Glycosylated hemoglobin (HbA1c). In addition to this, Thyroid Stimulating hormone (TSH) will also be estimated.

The individuals having impaired values for any of these tests (Fasting plasma glucose $100-125 \mathrm{mg} / \mathrm{dL}$, Glycosylated hemoglobin (5.7-6.4\%) will then undergo Oral Glucose Tolerance Test (OGTT) between 140 and $<200 \mathrm{mg} / \mathrm{dl}$, for further confirmation of pre-diabetic state.

Step 2: Training of Yoga and physical exercise procedures

The pre-diabetics so identified through community screening will participate in this step. They will be randomly allocated to either Yoga or exercise group. Their family history, medical \& surgical history, addictions, diet \&psychological stress will be documented. Dietary pattern of the participants will be recorded using Food Frequency Questionnaire, while the stress levels will be evaluated using Perceived Stress Scale. 
Following which, they will be randomly divided into 2 groups. The randomization will be done by computer generated randomized method in a 1:1 ratio. The method of block randomization will be used. One group will receive defined Yoga interventions, while other group will receive fixed program of physical exercises. As it is an open labelled study, no blinding mechanism will be followed.

Before carrying out the comparison, both groups will undergo a training session for a period of 1 month (4 weeks) under the supervision of a trained instructor. Since properly carried out Yoga procedures or exercises can only produce optimal effects, the training period is considered essential. During training period, the instructor will assess attendance, motivation and learning abilities of the participants.

The Yoga and exercise programs will be of 60 minutes duration, daily. The components of both programs will be as follows:

Yoga protocol ${ }^{10}$ -

Breathing practices $\quad 6 \mathrm{~min}$

Loosening exercises $\quad 10 \mathrm{~min}$

Yogasana $32 \mathrm{~min}$

Deep Relaxation Technique $\quad 6$ min

Pranayama 6 min

This protocol has been designed as per Swami Vivekananda Yoga Anusandhana Samsthana (SVYASA) diabetes yoga program. The detailing of all the individual components will be done to the participants.

Exercise protocol-

Warm up 5 min

Tread mill $15 \mathrm{~min}$

Cycling 15 min

Cross trainer exercise $\quad 15 \mathrm{~min}$

Abdominal exercise $\quad 10 \mathrm{~min}$

Both, the Yoga sessions and the exercise regime will be conducted at Bharati Vidyapeeth, College of Physical Education. There will be 2 batches - morning (6am-8am) and evening ( $6 \mathrm{pm}-8 \mathrm{pm})$ - made available to the participants. They may attend either of the two sessions, as per their convenience. There will be 6 sessions per week. 
The following details will be recorded from each participant pre- and post-training period.

1. Weight, height

2. Blood pressure, pulse rate

3. Body composition: Fat percentage, Muscle mass percentage using Bio-impedance method

4. Circumferential measures: waist, hip, neck, mid-arm, mid-thigh, abdomen

5. Muscle assessment

a.Muscle strength:

i. Hand grip strength

ii. Leg strength dynamometer

b. Muscle endurance: Push-ups, dips, pull-ups/arm hang, box jump test using standardized protocols

c. Muscle flexibility using flexometer/goniometer

6. Cardio respiratory fitness:

a. Metabolic equivalents (METs)using IPAQ (International Physical Activity Questionnaire)

b. Aerobic power $\left(\mathrm{VO}_{2} \mathrm{max}\right)$ by Queen's college step test for cardiac endurance

We expect that of 50 individuals will participate in Step 2 and at least 40 individuals in each group will enter in the next step.

Step 3: Interventional study of Yoga vis-à-vis exercise

The individuals who will successfully complete the training sessions will enter the Step 3 of the study. They will continue following the same program as per the assigned group (Yoga or exercise) for a period of 12 weeks. Like training period, there will be 6 sessions every week, thus 24 sessions per month. The participants need to attend minimum 18 sessions per month. In addition, individuals who will be unable to attend the sessions for more than 4 consecutive days, will be withdrawn from the study.

In addition to clinical and muscle assessment mentioned in Step 2, changes in the dietary pattern (if any) and psychological stress (using Perceived Stress Scale) will be done at this visit and then on monthly basis. Further, this step will involve blood investigations as listed below. All the investigations will be carried out before and after study, except FBG that will be done monthly. The parameters will be estimated using commercially available kits following manufacturer's instructions.

1. Glucose metabolism markers: FBG, Fasting Insulin, $\mathrm{HbA1c}$

2. Lipid profile: Total Cholesterol, Triglycerides, HDL, LDL, Serum Free Fatty Acids

3. Inflammatory marker (adipocytokine): Adiponectin

4. Muscle metabolism markers: 
- Enzymes: Creatine kinase, Lactate dehydrogenase, Alkaline phosphatase mitochondrial ATPase

- Protein: Myoglobins by ELISA method using kits

5. Oxidative stress: MDA, ORAC by spectrophotometric analysis.

During the study period, there will be counseling sessions for all participants every month regarding lifestyle modifications (diet, physical activity and behavior) to motivate them and keep their interest intact in the intervention programs. Apart from this, dietary advice will be given to the participants.

We expect $~ 30$ individuals from each group to complete the study.

\section{Outcome Measures-}

The outcome measures will be parameters related to muscle quality/functionality and glucose metabolism markers as explained earlier.

\section{Statistical Considerations}

Intention-To-Treat (ITT) analysis will be carried out i.e., data of all recruited participants will be considered. All evaluated parameters will be compared within group and between groups. The data will also be analyzed sex wise (male-female) and age wise (30- $\leq 40$ years and $>40-49$ years). The association of parameters related to muscles will be studied with the parameters of glucose metabolism. Age and sex are the expected confounding variables, which will be taken care of by adjusting data using statistical modeling. As we have adopted block randomization, interim analysis can be done. The statistical analysis is planned as per ITT, hence missing data will be handled by LOCF (Last Observation Carried Forward).

\section{Ethical Aspects}

This study was approved by the Institutional Ethics Committee, Bharati Vidyapeeth Deemed to be University, Pune on $4^{\text {th }}$ March, 2019 (IEC/2019/05). Further, the approval for amendment was received on $6^{\text {th }}$ July, 2019 (IEC/2019/35). Prospective registration of this study was done in 'The Clinical Trials Registry- India' on 15/05/2019 (CTRI/2019/05/019149).

While conducting the study, informed consent will be obtained from all eligible participants willing to participate in the study before any procedures. Each participant will be informed that participation in the trial is voluntary and that they are free to withdraw their participation, without justification, from the trial at any time without consequences and without affecting any professional responsibilities.

\section{Data safety and monitoring.plan}

There is very low risk posed to participants of this study. Thus, the data safety and monitoring will be done by the study team. The spontaneous adverse events will be documented for each participant in the CRF. The funding agency will be responsible for the overall monitoring of the data. 


\section{Trial Status}

The trial recruitment has begun on $4^{\text {th }}$ November 2019. The recruitment was stopped after $15^{\text {th }}$ March 2020 due to COVID-19 pandemic. We anticipate to complete the study by May 2022.

\section{Discussion}

The study aims to address one of the most prevalent and detrimental health issues i.e., pre-diabetes. The world staggers with the burden of DM and its complications. Since the pre-diabetic stage is reversible, it becomes crucial to understand more of its mechanisms and eventually management.

We have planned to test the effect of Yoga on insulin resistance and glucose uptake in skeletal muscles of pre-diabetic individuals. Though earlier studies have proven role of Yoga in achieving glycemic control and thereby reversing pre-diabetic stage, the mechanism behind this remains unexplored. ${ }^{11}$ Certain postulations such as stress reduction, action on Psycho-Neuro-Endocrine axis ${ }^{12}$ etc. have been put forward, however the effect on musculoskeletal system has not been elucidated. Since physical activities such as Asana are integral to Yoga, it is likely that they may have effect on intra-myocellular fat deposition that is seen in case of obesity, prediabetes and diabetes. Effect of physical exercise regimen has been well established on the fat deposition in muscles and its related consequences on muscle functionality. ${ }^{13}$ Hence, we selected exercise as a comparator in the present study.

One of the interesting feature of this study is the focus on lifestyle related interventions, which are cornerstone of lifestyle diseases. Apart from the therapeutic benefits in pre-diabetes, our study intends to impart awareness about pre-diabetes in community through screening campaigns and also to provide pre-diabetic individuals with active coping skills for reversing it. The other interesting feature of the study is its interdisciplinary nature, wherein the conventional clinical research methodology is applied to traditional science of Yoga. It was challenging in a way to draft a study protocol. Due to complex nature of interventions, defining various points of the protocol, especially eligibility criteria, compliance, withdrawal criteria, safety assessment etc. were tough. A major issue about the interventions like exercise and yoga is that they cannot be helpful if done in wrong manner. To avoid this, we included a training session of 1-month duration. This approach can help in protocol adherence as well as participant retention during intervention period.

It is expected that the present study will bridge the gap in existing knowledge about Yoga and will prove a step ahead towards evidence based yoga.

\section{Abbreviations}

BMI- Body Mass Index,

T2DM- Type 2 Diabetes mellitus, 
FBG- Fasting Blood Glucose,

ELISA- Enzyme-Linked Immunosorbent Assay,

MDA-Malondialdehyde,

ORAC- Oxidative Stress and Total Anti-Oxidant Capacity

\section{Declarations}

\section{Ethics approval and consent to participate-}

The study protocol and consent to participate were reviewed and approved by the Institutional Ethics Committee, Bharati Vidyapeeth Deemed to be University, Pune on 4th March, 2019 (IEC/2019/05). Further, the approval for amendment was received on 6th July, 2019 (IEC/2019/35). Written informed consent will be obtained from all study participants.

Consent for publication- Not Applicable

Availability of data and materials- Not Applicable

Competing interests-The authors declare that they have no competing interests.

Funding- This study is funded by Department of Science \& Technology, Government of India under 'Science and Technology in Yoga and Meditation' (SATYAM) scheme. The funding agency will be responsible for the monitoring the data only.

Authors' contributions- SB conceived the idea and actively contributed in protocol development, JK gave inputs from yoga-exercise and physiology perspective and SH drafted the protocol. All authors have read and approved the final manuscript for publication.

Acknowledgements- The authors acknowledge valuable ongoing support of Mrs. Anita Patil, Yoga instructor and Mr. Bapu Borotikar, Yoga expert. We would like to thank the members of Trial Steering Committee; Dr. Vijaya Pandit, Dr. Girish Tillu and Dr. Vaishali Deshmukh. We acknowledge Dr. Vedvati Bhapkar for her help in submitting the manuscript for publication.

\section{References}

1. Tripathy J, Thakur J, Jeet G, Chawla S, Jain S, Pal A et al. Prevalence and risk factors of diabetes in a large community-based study in North India: results from a STEPS survey in Punjab, India.

Diabetology \& Metabolic Syndrome. 2017;9(1).

2. Keerthi GS, Pal P, Pal GK, Sahoo JP, Sridhar MG, Balachander J. Effect of 12 Weeks of Yoga Therapy on Quality of Life and Indian Diabetes Risk Score in Normotensive Indian Young Adult Prediabetics 
and Diabetics: Randomized Control Trial. J Clin Diagn Res. 2017;11(9):CC10-CC14. doi:10.7860/JCDR/2017/29307.10633

3. Kanat M, DeFronzo RA, Abdul-Ghani MA. Treatment of prediabetes. World J Diabetes. 2015;6(12):1207-1222. doi:10.4239/wjd. v6.i12.1207

4. Dide Rast Sh., Hojjati Z., Shabani R. The Effect of Yoga Training on Blood Glucose, Insulin and Resting Heart Rate in Type II Diabetic Females. Res. J. Sport. Sci.;2014; 2 (1);15-21

5. DeFronzo R, Tripathy D. Skeletal Muscle Insulin Resistance Is the Primary Defect in Type 2 Diabetes. Diabetes Care. 2009;32(suppl_2): S157-S163.

6. Büssing A, Michalsen A, Khalsa S, Telles S, Sherman K. Effects of Yoga on Mental and Physical Health: A Short Summary of Reviews. Evidence-Based Complementary and Alternative Medicine. 2012; 2012:1-7.

7. Chimkode SM, Kumaran SD, Kanhere VV, Shivanna R. Effect of yoga on blood glucose levels in patients with type 2 diabetes mellitus. J Clin Diagn Res. 2015;9(4):CC01-CC3. doi:10.7860/JCDR/2015/12666.5744

8. Dasappa H, Fathima F, Prabhakar R. Effectiveness of yoga program in the management of diabetes using community health workers in the urban slums of Bangalore city: A non-randomized controlled trial. Journal of Family Medicine and Primary Care. 2016;5(3):619.

9. Pereira R, Moura L, Muñoz V, Silva A, Gaspar R, Ropelle E et al. Molecular mechanisms of glucose uptake in skeletal muscle at rest and in response to exercise. Motriz: Revista de Educação Física. 2017;23(spe).

10. Srikanta, S., Nagarrathna,N. and Nagendra, H.:'Yoga for Diabetes'. Vivekananda Yoga Research Foundation, Swami Vivekananda Prakashana. Banglore. 2003

11. Raveendran AV, Deshpande A, Joshi SR. Therapeutic Role of Yoga in Type 2 Diabetes. Endocrinol Metab (Seoul). 2018;33(3):307-317. doi:10.3803/EnM.2018.33.3.307

12. Singh VP, Khandelwal B, Sherpa NT. Psycho-neuro-endocrine-immune mechanisms of action of yoga in type II diabetes. Anc Sci Life. 2015;35(1):12-17. doi:10.4103/0257-7941.165623

13. Spriet, L.L. New Insights into the Interaction of Carbohydrate and Fat Metabolism During Exercise. Sports Med. 2014. 44, 87-96. https://doi.org/10.1007/s40279-014-0154-1

\section{Figures}




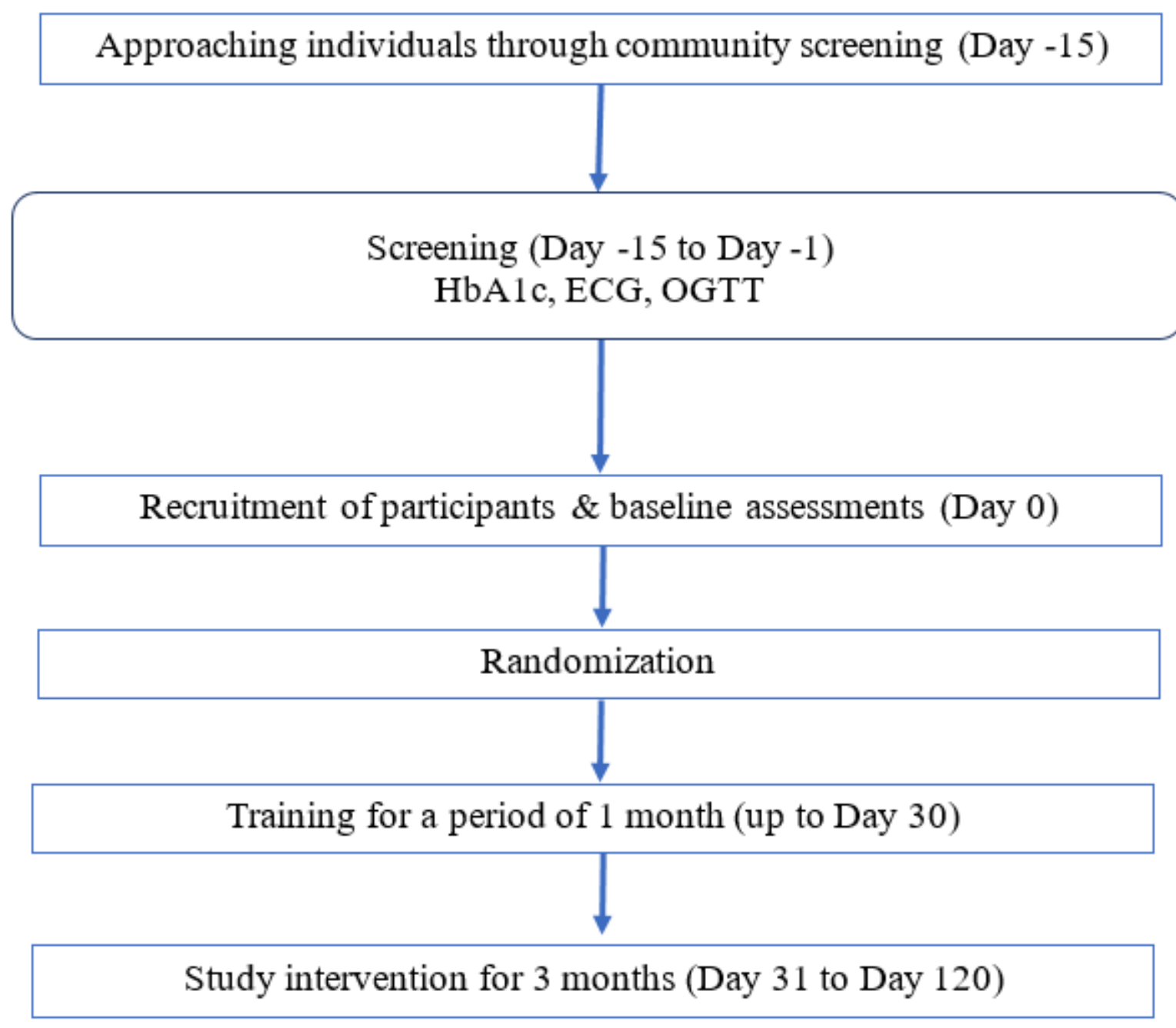

Figure 1

Participant timeline

\section{Supplementary Files}

This is a list of supplementary files associated with this preprint. Click to download.

- SPIRITchecklistsuppl.file1030221.doc

- ICDsuppl.file2030221.pdf 